Research Paper

\title{
Detection of High-Risk Atherosclerotic Plaques with Ultrasound Molecular Imaging of Glycoprotein Ilb/llla Receptor on Activated Platelets
}

\author{
Shengcun Guo, Shuxin Shen, Junfen Wang, He Wang, Meiyu Li, Ying Liu, Fanfan Hou, Yulin Liao, \\ Jianping Bin ${ }^{凶}$
}

State Key Laboratory of Organ Failure Research, Department of Cardiology, Nanfang Hospital, Southern Medical University, Guangzhou, China.

\begin{abstract}
$\triangle$ Corresponding author: Jianping Bin, State Key Laboratory of Organ Failure Research, Department of Cardiology, Nanfang Hospital, Southern Medical University, 1838 North Guangzhou Avenue, Guangzhou 510515, China. E-mail: jianpingbin@126.com / jianpingbin@hotmail.com. Tel: (+86) 20-61642365; mobile: (+86) 18680488488; fax: (+86) 20-87712332

(C) 2015 Ivyspring International Publisher. Reproduction is permitted for personal, noncommercial use, provided that the article is in whole, unmodified, and properly cited. See http://ivyspring.com/terms for terms and conditions.
\end{abstract}

Received: 2014.07.01; Accepted: 2014.12.28; Published: 2015.02.05

\begin{abstract}
Objective: Ultrasound molecular imaging (UMI) of glycoprotein (GP) IIb/Illa receptor on activated platelets offers a unique means of identifying high-risk atherosclerosis. We hypothesized that contrast-enhanced ultrasound with microbubbles (MBs) targeted to GP Ilb/llla could be used to detect and quantify activated platelets on the surface of advanced plaques.

Methods and Results: A mouse model of advanced atherosclerosis was generated by maintaining apolipoprotein E-deficient $\left(\mathrm{ApoE}^{-/-}\right)$mice on a hypercholesterolemic diet $(\mathrm{HCD})$. The three other experimental groups consisted of $\mathrm{ApoE}^{-/-}$and wild-type (C57BL/6) mice fed a normal chow diet and C57BL/6 mice on an HCD diet. Plaque formation was confirmed by histological and immunohistochemical methods using light, fluorescence, and electron microscopy. Mice were injected with a lipid MB-conjugated cyclic Arg-Gly-Asp peptide or nonspecific control peptide, and the abdominal aorta was examined by UMI. The accumulation of GP IIb/llla and activated platelets on the surface of atherosclerotic plaques was highest in the $A p o E^{-1-}+\mathrm{HCD}$ group, followed by $\mathrm{ApoE}^{-1-}+$ chow, C57BL/6+HCD, and C57BL/6+chow groups $(P<0.05)$. Notably, GP Ilb/llla expression was associated with the vulnerability index and necrotic center/fiber cap ratio $(P<0.05)$, and contrast video intensity from adhered cyclic Arg-Gly-Asp-modified MBs (MB-cRGDs) was correlated with GP Ilb/llla expression on the plaque surface $(P<0.05)$.

Conclusion: GP IIb/Illa of activated platelets on the atherosclerotic endothelium is a biomarker for high-risk plaques that can be quantified by UMI using MB-cRGDs, providing a noninvasive means for detecting high-risk plaques and preventing acute cardiovascular events.
\end{abstract}

Key words: Atherosclerosis; Microbubbles; Molecular imaging; Platelets; Glycoprotein IIb/IIIa

\section{Introduction}

Identifying high-risk atherosclerotic plaques is necessary for guiding the management and preventing the occurrence of severe cardiovascular events [1]. A variety of noninvasive molecular imaging approaches can detect atherosclerosis on a subcellular level and identify suspected vulnerable plaques including ultrasound molecular imaging (UMI), which is widely available, inexpensive, and well-suited to high-throughput evaluation of atherosclerotic phenotypes $[2,3]$. 
UMI can be used to detect molecular targets on the surface of activated platelets and endothelial cells that are accessible to microbubbles (MBs), including activated von Willebrand factor (vWF) for platelet adhesion [4,5], vascular cell adhesion molecule-1 and P-selectin for inflammation [5-7], and vascular endothelial growth factor receptor-2 and interleukin-8 for neovascularization $[8,9]$, all of which are expressed on the endothelial surface. To date, there are no reports of an atherosclerotic profile of activated platelets and thrombosis obtained by UMI. While these play an important role in the progression of atherosclerotic lesions [10, 11], more often, atherothrombosis is asymptomatic and insidious [11], and can be a warning sign for high-risk plaques which can potentially be detected by UMI of activated platelets.

The glycoprotein (GP) IIb/IIIa complex, also known as aIIb $\beta 3$ integrin, is the major receptor expressed on the surface of activated platelets and is essential for their interactions with other activated platelets and adjacent cells in atherothrombosis [11-13]. Platelet aggregation and GP IIb/IIIa expression on the circulating platelet surface or atherosclerosis were found to be higher in acute coronary syndrome or unstable angina than in stable angina or healthy volunteers [14-18]. Thus, the GP IIb/IIIa receptor is a potential marker for imaging aggregated platelets in atherosclerotic plaques. However, data on the correlation between GP IIb/IIIa on platelets in advanced atherosclerotic lesions and plaque severity are limited, and it is unclear whether GP IIb/IIIa on activated platelets can serve as a biomarker for high-risk plaques.

Peptides containing the Arg-Gly-Asp (RGD) sequence are highly adhesive for GP IIb/IIIa $[19,20]$. Cyclic (c) RGD has 30-fold greater affinity for the GP $\mathrm{IIb} / \mathrm{III}$ a complex than the linear form [21, 22], which is particularly advantageous for binding under the conditions of rapid blood flow that occur in atherosclerotic arteries [23]. We recently developed a cRGD-modified MB (MB-cRGD) that is capable of binding to GP IIb/IIIa on activated platelets and thrombi in large arteries [24, 25]. The current study examined whether GP IIb/IIIa receptors on activated platelets that are adhered and aggregated on the endothelium can serve as a biomarker of atherosclerotic plaque instability, and can be identified and quantified by contrast-enhanced ultrasound using MB-cRGDs.

\section{Materials and Methods}

\section{Animal models and experimental protocol.}

The study protocol was approved by the Animal Research Committee at Southern Medical University, and all procedures were performed in accordance with the Institutional Guidelines for Animal Research and complied with the Guide for the Care and Use of Laboratory Animals published by the United States National Institutes of Health. Four groups of mice were used in this study: apolipoprotein E-deficient $\left(\mathrm{ApoE}^{-/-}\right)$mice purchased from the Vital River Laboratory Animal Technology Co. Ltd. (Beijing, China) and maintained on a hypercholesterolemic diet (HCD, containing $21 \%$ fat and $0.15 \%$ cholesterol by weight) (ApoE $\left.{ }^{-/-}+\mathrm{HCD} ; \mathrm{n}=30\right) ; \mathrm{ApoE}^{-/-}$mice fed a normal chow diet (ApoE ${ }^{-/-+}$chow; $\mathrm{n}=18$ ); wild-type $\mathrm{C} 57 \mathrm{BL} / 6$ mice on an HCD (C57BL/6+HCD; $n=18)$; and wild-type mice fed normal chow (C57BL/6+chow; $\mathrm{n}=18$ ). Mice were maintained on their respective diets for 30 weeks starting at 6 weeks of age according to a previous study [26], and then 6 mice in each group were randomly selected for the UMI and histopathological examination, 6 other mice in each group were randomly selected for injection with platelets labeled with the fluorescent dye calcein-AM and examination by fluorescence microscopy, and 12 mice randomly selected from the ApoE-/-+HCD group served as negative controls, with half administered calcein-AM only and the other half (autofluorescence control group) administered phosphate-buffered saline (PBS) without calcein-AM. The remaining 6 mice in each group were used to perform UMI blocking experiments (Fig.1). Supplementary in vitro experiments were carried out to evaluate the potential mechanism of GP IIb/IIIa to serve as a biomarker of vulnerable plaques (see Supplemental Materials for details).

\section{MB preparation.}

The GP IIb/IIIa-targeted and negative control MBs (MB-cRGD and MB-CON, respectively) were generated by conjugating $\mathrm{MBs}$ to cyclic Arg-Gly-Asp-D-Phe-Cys (molecular weight $=578.65$, $\mathrm{C}_{24} \mathrm{H}_{34} \mathrm{~N}_{8} \mathrm{O}_{7} \mathrm{~S}$ ) and cyclic Arg-Ala-Asp-D-Phe-Val (molecular weight $=588.67, \mathrm{C}_{27} \mathrm{H}_{40} \mathrm{~N}_{8} \mathrm{O}_{7}$ ) peptides, respectively, as previously described [24]. The binding characteristics of the MBs have been previously reported [24]. MB-CON and MB-cRGD were characterized with a Coulter counter (Beckman Coulter Inc., Brea, CA, USA) to determine the MB size and concentration (see Supplemental Materials for details). After counting, the MBs were diluted with saline to a concentration of $1 \times 10^{7}$ and their in vivo half-life was measured (see Supplementary Materials for details). Peptides were synthesized by Peptides International Inc. (Louisville, KY, USA).

\section{Attachment of fluorescence-labeled plate- lets to the aorta.}

Freshly isolated platelets were obtained from 
whole blood collected from normal healthy C57BL/6 mice and washed twice with PBS. The platelets were incubated with calcein AM $(300 \mathrm{ng} / \mathrm{ml}$ in PBS; Invitrogen, Carlsbad, CA, USA) for 15 min in the dark as previously described [25]. Fluorescence-labeled platelets were injected into four different mouse groups through the tail vein $(n=6$ each); with an equivalent amount of calcein-AM without platelets injected into the negative control mice $(n=6)$ and PBS without calcein-AM injected into the autofluorescence control ApoE ${ }^{-/-}+\mathrm{HCD}$ mice $(\mathrm{n}=6)$. Animals were sacrificed $15 \mathrm{~min}$ after injection, and the aorta was harvested and immediately frozen in optimum cutting temperature medium. The tissue was sectioned on a cryostat, visualized at $480 \mathrm{~nm}$ under an epifluorescence microscope, and imaged with a C150L charge-coupled device camera (Pixera, Santa Clara, CA, USA).

\section{Detection of activated platelets and athero- thrombus by electron microscopy (EM).}

Abdominal aorta tissue samples for the UMI study were fixed in situ with $2.5 \%$ glutaraldehyde, and a subset of these was prepared for EM following a standard procedure. The luminal surface was observed by scanning EM (S-3000N; Hitachi, Tokyo,

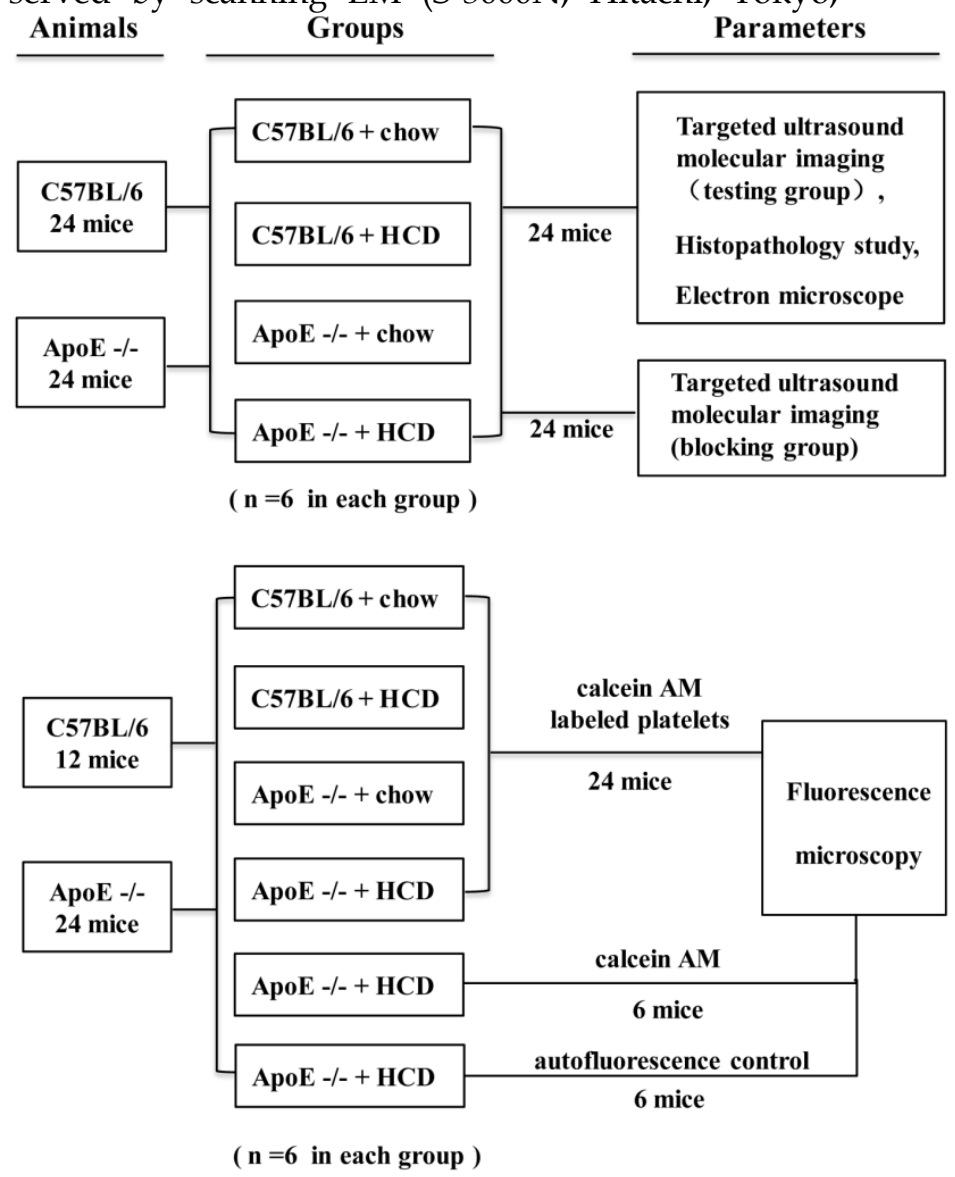

Figure 1: Illustration of the experimental protocol.
Japan) operated at $20 \mathrm{kV}$. Platelets adhered to the surface of the vessel lumen were quantified by counting the average number of platelets over $10 \mathrm{op}-$ tical fields $(25.2 \times 25.2 \mathrm{~mm}$ per field). Transmission EM (Tecnai G2Spirit; Fei, Hillsboro, OR, USA) was used at $80 \mathrm{kV}$ to examine activated platelets in the tissue of ApoE ${ }^{-/-}+\mathrm{HCD}$ mice.

\section{Histology and immunohistochemistry.}

Samples of the abdominal aorta for histopathologic examination were obtained from all 24 mice used in the UMI study. Hematoxylin and eosin and Masson's trichrome (MST-8003; Matxin Labs Pvt. Ltd., Bangalore, India) staining and immunohistochemistry were performed on paraffin-embedded serial sections cut at a thickness of $4 \mu \mathrm{m}$. Immunohistochemistry was performed using a rabbit polyclonal primary antibody against mouse a-smooth muscle actin (a-SMA), cluster of differentiation (CD)68, or GP IIb integrin (ab5694, ab125212, and ab63983, respectively; all from Abcam, Cambridge, MA, USA) to label smooth muscle cells (SMCs), macrophages, and platelets, respectively.

\section{Plaque quantification based on histopathologic indicators.}

Five representative serial sections each of the proximal, intermediate, and distal ends of the abdominal aorta were selected from each animal. Lipid deposition and collagen fiber content were measured by planimetry [27] and expressed as a percentage of total plaque area. The area of positive immunoreactivity was quantified using Image-Pro Plus (Media Cybernetics, Rockville, MD, USA) and expressed as a percentage of the total area of the plaque or vessel wall. The SMC and macrophage contents of plaque were quantified as percentages of positive to total plaque area. The GP IIb/IIIa content of plaques was measured by two methods: as percentages of GP IIb/IIIa expression in the plaque and of GP IIb/IIIa coverage of the endothelium, as previously described [28]. Histopathologic indicators (a-SMA, CD68, and GP IIb/IIIa expression) were quantified at three selected sites and averaged per section per site.

All measurements and analyses of UMI, scanning EM, histology, and immunolabeling data were performed by individuals who were blinded to the experimental design. The necrotic center/fiber cap (NC/FC) ratio was measured, and plaque vulnerability index was calculated using the formula $[29,30]$ : vulnera- 
bility index = (lipid deposit and macrophages) $/$ (collagen fibers and SMCs).

\section{UMI of atherosclerotic plaques.}

UMI was performed on 24 mice using a Sequoia ultrasound system with a high-frequency 15L7 linear-array probe secured in place using Cadence contrast pulse sequencing with contrast harmonic imaging (Siemens Medical Systems, Mountain View, CA, USA). The abdominal aorta was imaged by conventional ultrasound at $15 \mathrm{MHz}$ to optimize the imaging plane along the longitudinal axis at a centerline frequency of $7 \mathrm{MHz}$ and mechanical index of 0.18 . Gain settings were adjusted to and maintained at levels just below the visible noise speckle. Real-time images of the aorta were acquired at an index of 0.18 for $10 \mathrm{~min}$ after intravenous injection of $1 \times 10^{6} \mathrm{MB}-\mathrm{cRGD}$ or $\mathrm{MB}-\mathrm{CON}$ in a total volume of $0.1 \mathrm{ml}$ in a random order; the mechanical index was transiently increased to 1.0 for $3 \mathrm{~s}$ to destroy adhered MBs, and post-destruction images were acquired at an index of 0.18 to obtain the background signal as previously described $[4,6,31]$. To identify the signal associated with retained MBs, three post-destruction contrast frames representing the signal from circulating MBs were averaged and digitally subtracted from three averaged pre-destruction frames using Yabko MCE2.7 image analysis software (University of Virginia, Charlottesville, VA, USA) and then color-coded; background-subtracted video intensity (VI) was measured from a region of interest encompassing the abdominal aorta.

To demonstrate the high affinity of MB-cRGD for the GP IIb/IIIa receptor in vivo, a competitive inhibition experiment was performed using 24 mice. Eptifibatide (integrilin), a GP IIb/IIIa antagonist used in clinical practice, was injected at a dose of $1.8 \mu \mathrm{g} / \mathrm{g}$ to saturate integrins followed by injection of MB-CRGD or MB-CON and performance of contrast enhanced ultrasound.

\section{Statistical analysis}

Data were analyzed using SPSS v.13.0 (SPSS Inc., Chicago, IL, USA) and are presented as the mean \pm standard deviation. Comparisons between two or four groups were performed with the independent-samples $t$-test and one-way analysis of variance, respectively. A Bonferroni correction was calculated for multiple comparisons of continuous variables with equal variances (determined by the homogeneity of variance test), while Dunnett's T3 test was used for multiple comparisons without equal variances. Spearman rank correlations were used to assess the linear correlation between selected variables. A $P$ value $<0.05$ was considered statistically significant.

\section{Results}

Fluorescent platelets attach to the endothelium of atherosclerotic lesions.

Freshly isolated platelets were labeled with fluorescent dye (Fig. 2A, B), and activated platelets adhering to the atherosclerotic lesions were observed by epifluorescence microscopy (Fig. 2D), with the highest numbers observed after injection of fluorescence-labeled platelets into the $\mathrm{ApoE}^{-/-}+\mathrm{HCD}$ group (Fig. 2Dd, i) followed by $\mathrm{ApoE}^{-/-+\mathrm{chow}}$, C57BL/6+HCD and C57BL/6+chow groups $(P<0.05$; Fig. 2E). There were no fluorescence-labeled platelets observed on the endothelium of the negative control group (Fig. 2De, j), or on atherosclerotic plaque in the autofluorescence control group (Fig. 2C).

\section{Activated-platelets adhere and aggregate on atherosclerotic plaque.}

The aggregation of platelets on the luminal surface of the endothelium was examined by scanning EM (Fig. 3A). In ApoE ${ }^{-/-+H C D}$ group, much more platelets were recruited in the atherosclerotic lesions, while some of them aggregated, suggesting an adhering to each other (Fig. 3Ad, h). Similar to results obtained with the fluorescent probe, the highest number of platelets was observed in the Ap$\mathrm{oE}^{-/-}+\mathrm{HCD}$ group, followed by $\mathrm{ApoE}^{-/-+}$chow, C57BL/6+HCD, and C57BL/ $6+$ chow groups $(P<0.05$; Fig. 3B). Because the size of platelet is variable (diameter may differ from 1-8 $\mu \mathrm{m}$ ) [32], thus it is not reliable enough to identify platelet according to its size by using scanning EM. We further confirmed the structure of platelet by transmission EM. We noted that the platelets could be recruited in the atherosclerotic plaque (Fig. 3Ca) or bind to endothelial cells (Fig. $3 \mathrm{Cb}$ ), and the microparticles, a feature structure of platelet, could be clearly observed in the platelets (Fig. $3 \mathrm{Cc}, \mathrm{d})$. Furthermore, thrombi - which are enriched in platelets and contain a large number of red blood cells (RBCs) and an abundance of fibrin - were detected at the site of vascular injury (Fig. 3D).

\section{Immunohistochemical and histopathological indictors of atherosclerosis.}

GP IIb/IIla expression in abdominal aortic plaques was assessed in the four experimental groups (Fig. 4A); GP IIb/IIIa coverage of the endothelium was highest in the ApoE $\mathrm{E}^{-/-}+\mathrm{HCD}$ group, followed by ApoE $E^{-/-+}$chow, C57BL/6+HCD, and C57BL/6+chow groups $(P<0.05$; Fig. $4 \mathrm{~B})$, and a similar expression pattern was observed in plaques (Fig. 4C). Interestingly, GP IIb/IIIa coverage of the endothelium was correlated with GP IIb/IIIa expression in plaques (Fig. 4D), and GP IIb/IIIa-rich thrombi were observed at the site of vascular injury (Fig. 4E). 
A
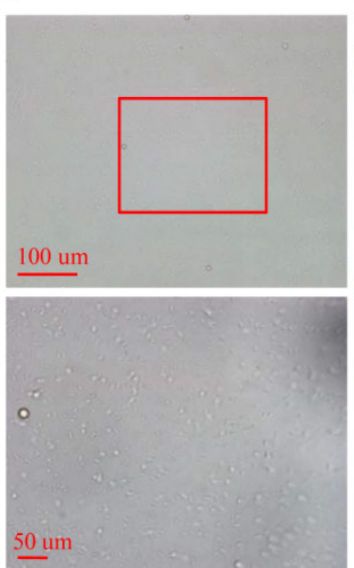

B
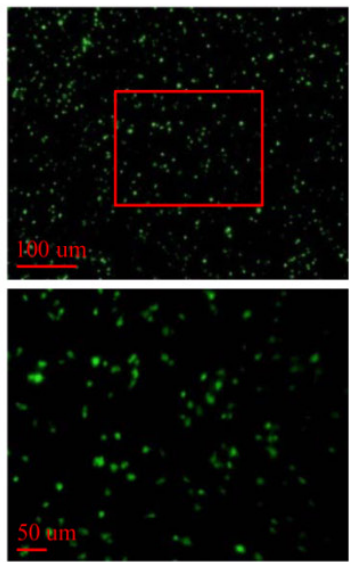

C

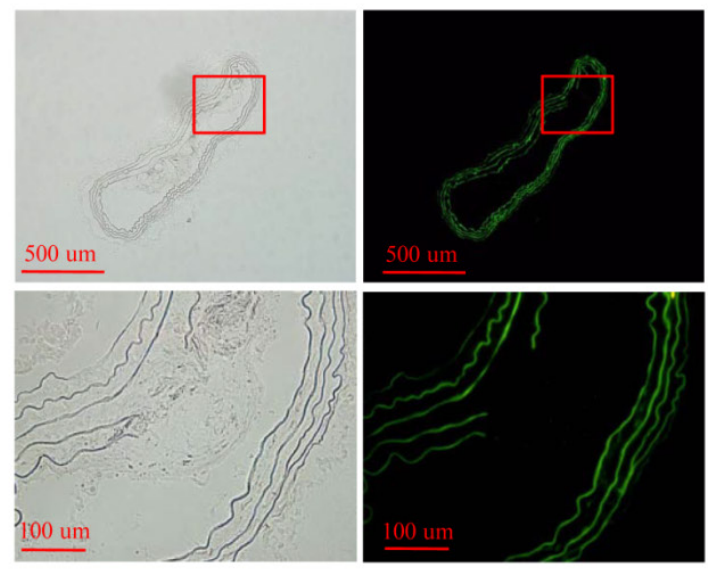

D

calcein AM-labeled platelets

calcein AM
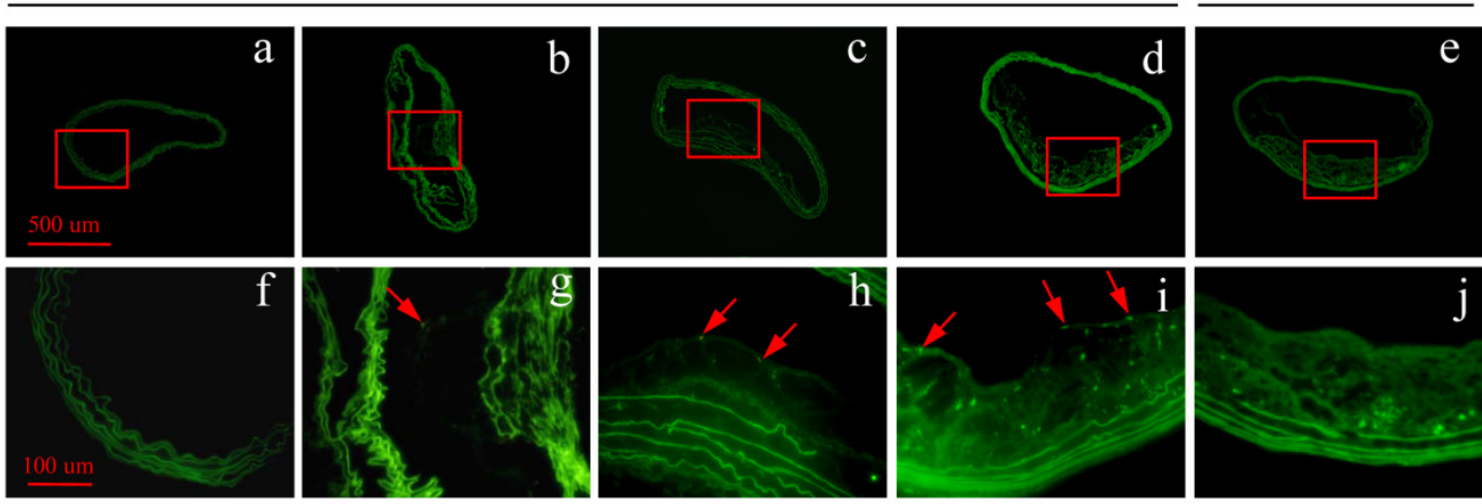

C57BL/6

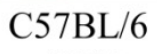

+chow

$+\mathrm{HCD}$

ApoE-/-

+chow
ApoE-/-

$+\mathrm{HCD}$

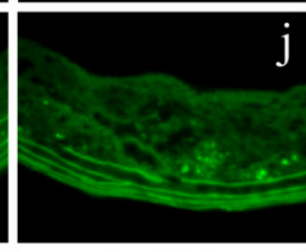

ApoE-/-

$+\mathrm{HCD}$

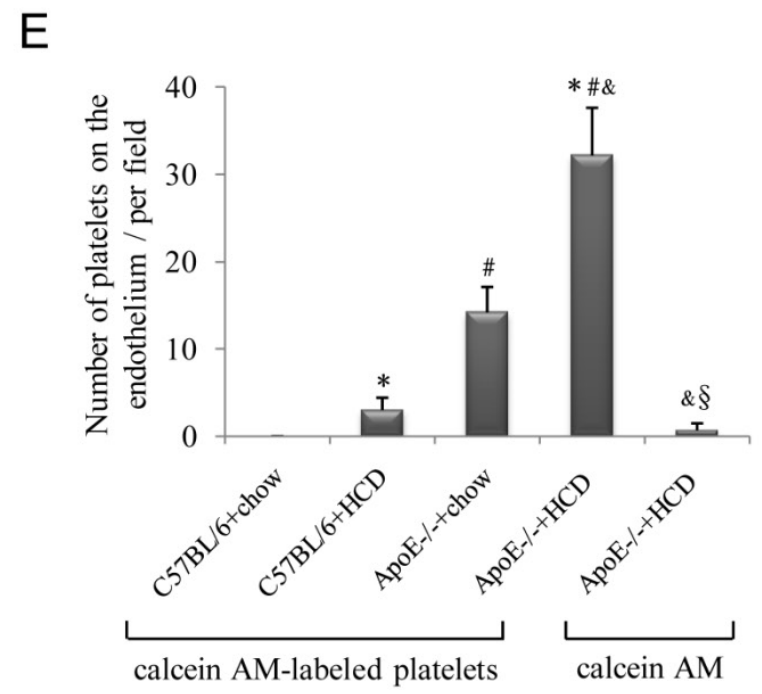

Figure 2: Attachment of fluorescently labeled platelets to the atherosclerotic endothelium. (A, B) Fluorescently labeled platelets were visualized by light $(\mathbf{A})$ and epifluorescence (B) microscopy. Platelets were injected into wild-type (C57BL/6) or ApoE-deficient (ApoE ${ }^{-}{ }^{-}$) mice maintained on chow or an $\mathrm{HCD}$. (C) Representative image of an aorta from the autofluorescence control group. (D) Representative images of the aorta of mice from the four experimental groups (a-d;

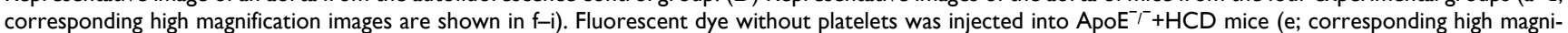
fication image is shown in j). Fluorescently labeled platelets aggregated on the endothelium of the aorta (red arrows in g-i). (E) Quantitative analysis of fluorescently labeled platelets ( $\mathrm{n}=6$ per group). Data represent mean \pm standard deviation. ${ }^{*} P<0.05$ vs. $C 57 B L / 6+$ chow, $\# P<0.05$ vs. $C 57 B L / 6+H C D, \& P<0.05$ vs. ApoE ${ }^{-}-+$chow, $\S P<0.05$ vs. ApoE ${ }^{-\top}+\mathrm{HCD}+$ platelets. 
A
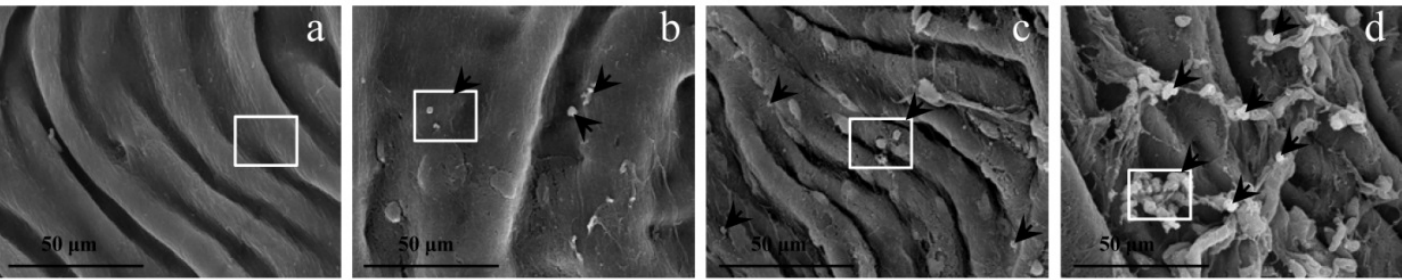

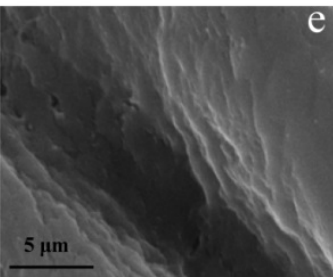

C57BL/6+chow

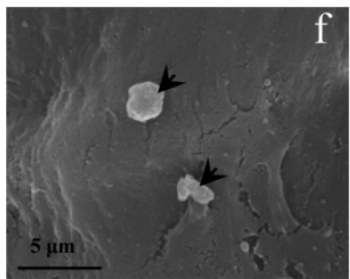

C57BL/6+HCD

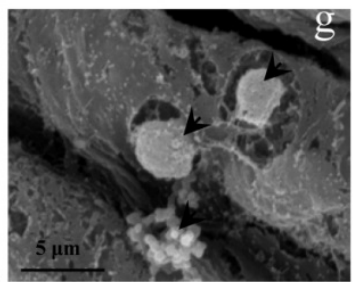

ApoE-/-+chow

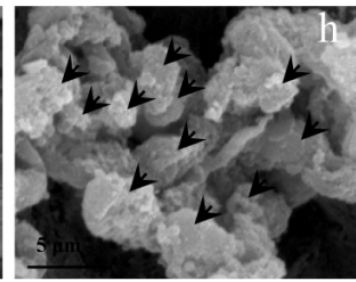

ApoE-/-+HCD
B

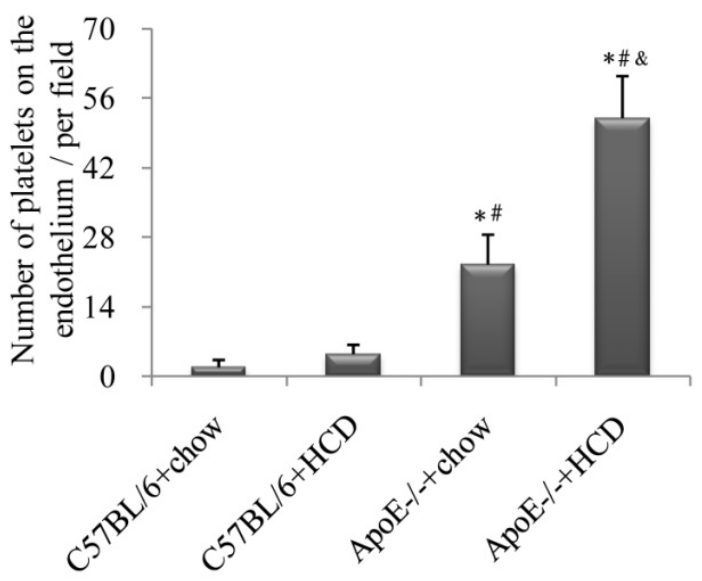

$\mathrm{D}$

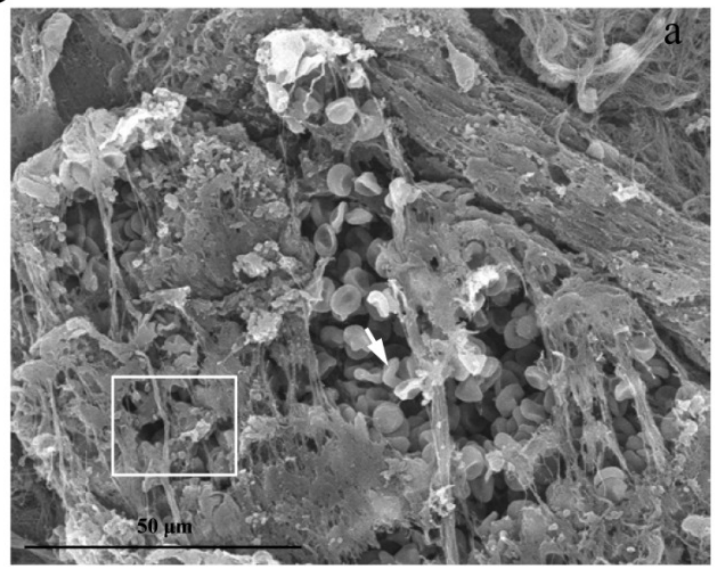

C
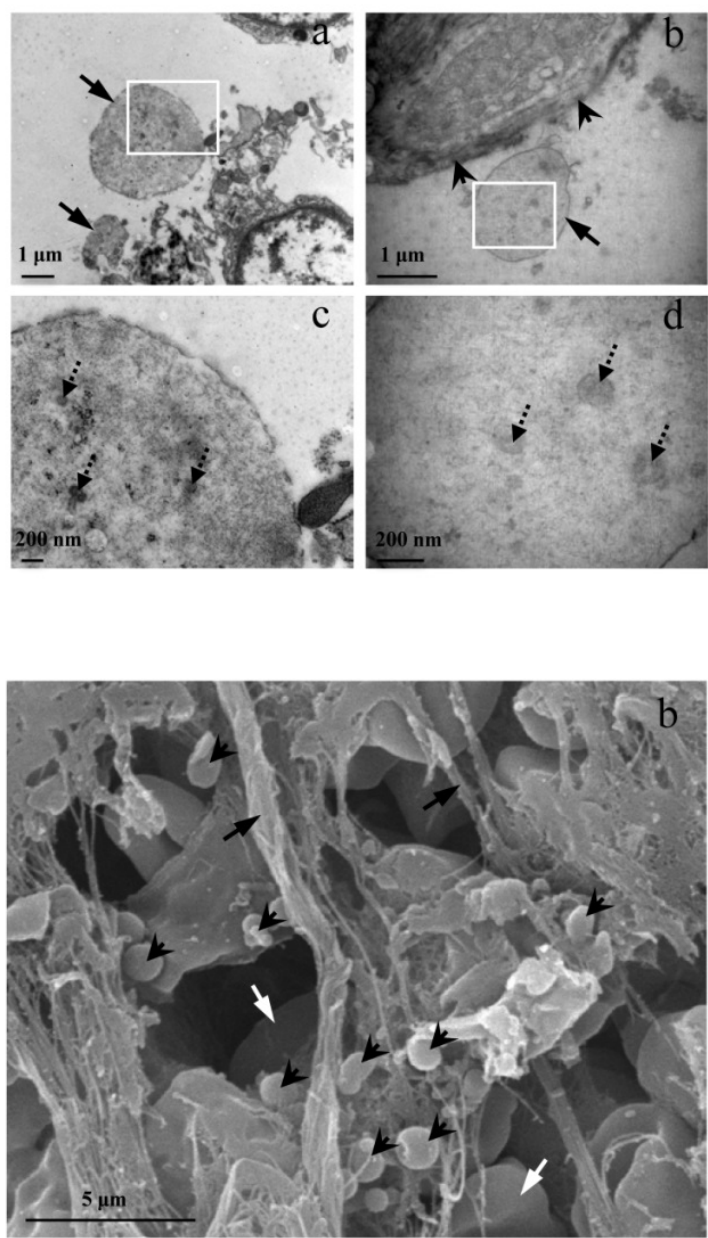

Figure 3: Platelet adhesion and aggregation on the endothelium of atherosclerotic plaques. (A) Platelets (arrowheads) adhering to the site of atherosclerotic lesions was observed by scanning EM. Panels from e-h are the magnified images of the corresponding white boxes in panels from a-d. Panel $h$ shows the activated platelets binding to each other. The number of platelets was markedly increased in ApoE---+HCD group (panel d). (B) Quantitative analysis of platelets based on scanning EM micrographs in panel $A$ ( $n=6$ per group), ${ }^{*} P<0.05$ vs. C57BL/6+chow, $\# P<0.05$ vs. C57BL/6+HCD, $\& P<0.05$ vs. ApoE ${ }^{-}{ }^{-}+$chow. (C) Representative images of recruited platelets from abdominal aorta in $A \mathrm{PoE}^{-} l^{-}+\mathrm{HCD}$ mice observed by transmission $\mathrm{EM}$ for structure confirmation of platelets. Panels $\mathrm{c}$ and $\mathrm{d}$ are the magnified images of the corresponding white boxes in panels a and b. Panel a: Two recruited platelets (arrows); Panel b shows the endothelium (arrowheads) and a platelet (arrow); Panel $\mathrm{c}$ and d show microparticles (dot arrows) in platelets. (D) Representative images of abdominal aorta from ApoE ${ }^{-}++\mathrm{HCD}^{-}$mice observed by scanning EM, in which thrombi enriched in platelets (arrowheads), red blood cells (white arrows), and fibrin (black arrows) are shown. 
A
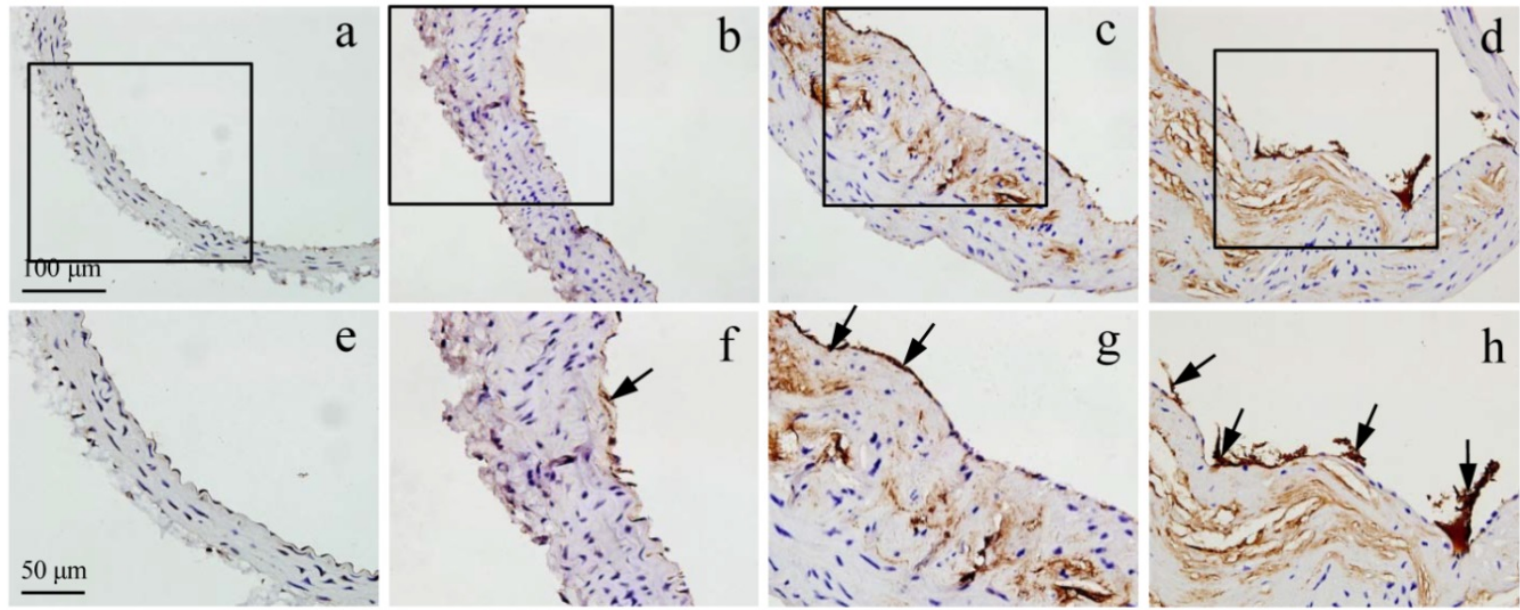

\section{C57BL $/ 6$}

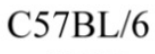

+chow

$+\mathrm{HCD}$

ApoE-/-
+ chow

ApoE-/-

$+\mathrm{HCD}$

B

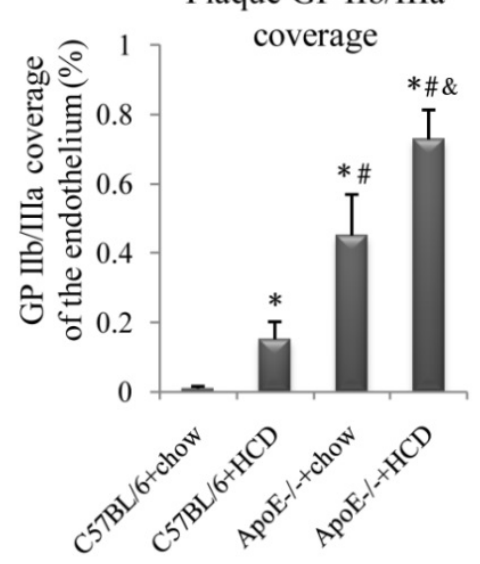

Plaque GP IIb/IIIa

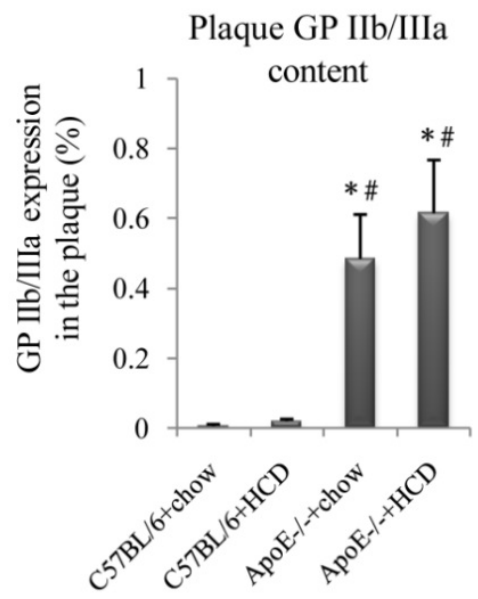

D

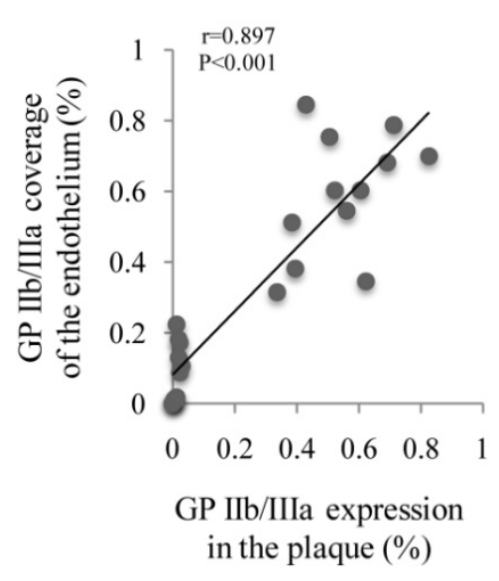

E
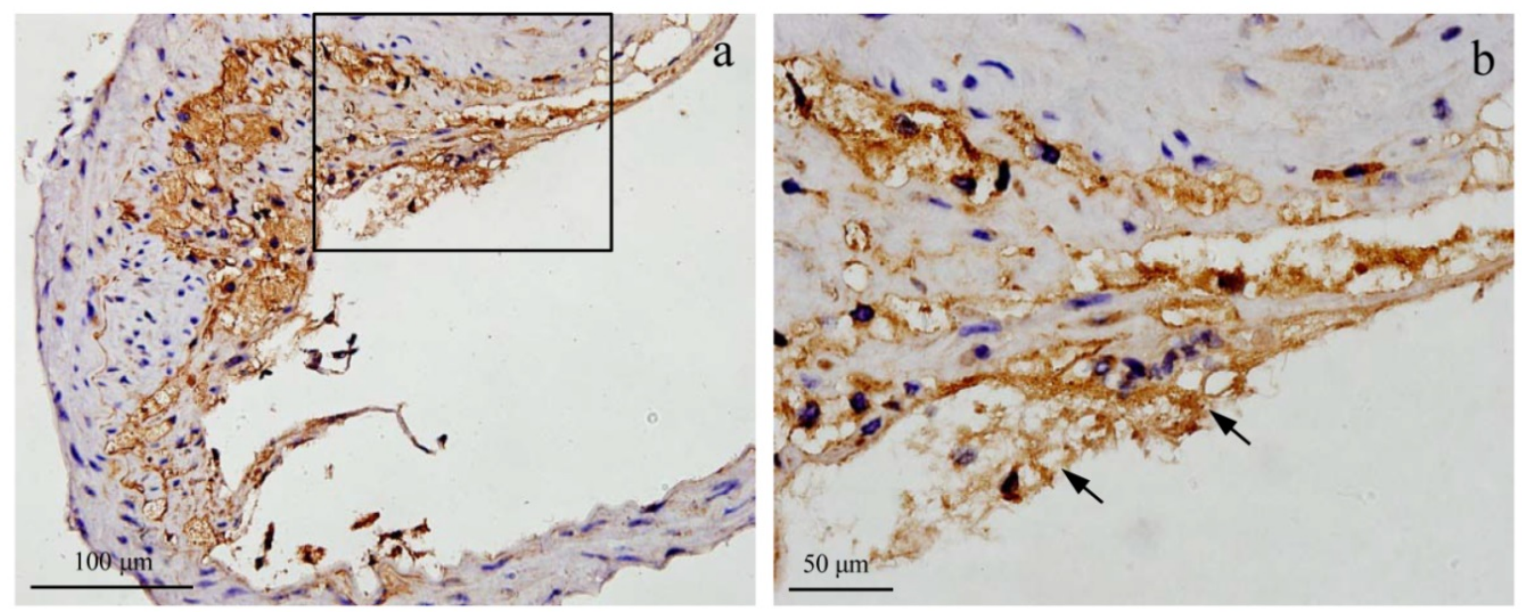

Figure 4: Immunohistochemical detection of glycoprotein (GP) IIb/IIla. (A) Representative images of GP IIb/Illa labeling in plaques (a-d) and the endothelium (e-h, arrows) of the aorta. Experimental groups are as described in the Figure 2 legend. Quantification for (B) GP Ilb/llla coverage of the endothelium and (C) total plaque GP Ilb/Illa content are shown ( $n=6$ per group). (D) Correlation between GP Ilb/llla coverage of the endothelium and GP IIb/IIla expression in plaques. (E) Representative images of thrombi enriched with GP Ilb/llla (b, arrows) in an $\mathrm{ApoE}^{-}{ }^{-}+\mathrm{HCD}$ mouse. 
Hematoxylin and eosin and Masson's trichrome staining, as well as a-SMA and CD68 immunolabeling of abdominal aortic plaques (Fig. 5A), revealed the highest percentage of macrophage-positive to total plaque area, NC/FC, and vulnerability index in Ap$\mathrm{oE}^{-/-}+\mathrm{HCD}$ mice, followed by $\mathrm{ApoE}^{-/-+}$chow, C57BL/6+HCD, and C57BL/6+chow mice $(P<0.05$; Fig. $5 \mathrm{C}-\mathrm{E})$. In contrast, the percentage of $\mathrm{SMC}$ to total plaque area showed the opposite trend (Fig. 5B). Notably, GP IIb/IIIa coverage of the endothelium and expression in the whole plaque correlated with plaque indicators such as vulnerability index and NC/FC (Fig. 5F-I).

\section{Endothelial expression of vWF is induced by tumor necrosis factor- $\alpha$ (TNF- $\alpha$ ) and GP IIb/ IIla expression on platelet is induced by vWF.}

To test the effect of inflammatory stimulation on endothelia, human umbilical vein endothelial cell (HUVECs) were stimulated with various concentrations of TNF- $a$ and vWF expression was assessed. We found that TNF-a-induced vWF expression on HUVECs as well as vWF-induced GP IIb/IIIa expression on platelets was dose-dependent (Fig. 6A-D). The specificity of the immunoreactivity was confirmed by the absence of fluorescence in HUVECs or platelets incubated with secondary antibodies alone (Fig. 6A, C control). Hence, GP IIb/IIIa functions as a biomarker of vulnerable plaques as a result of inflammation and vWF activation, in accordance with previous findings [33].

\section{Targeted imaging of atherosclerotic plaques.}

Background-subtracted VI (Fig. 7A) for MB-cRGD and MB-CON were comparable in both the testing and blocking groups in C57BL/6+chow mice, but was significantly higher for MB-cRGD compared to MB-CON in the other three testing groups, with the highest value observed for $\mathrm{ApoE}^{-/-+\mathrm{HCD}}$ followed by $\mathrm{ApoE}^{-/-+}$chow and $\mathrm{C} 57 \mathrm{BL} / 6+\mathrm{HCD}$ groups. Treatment with Eptifibatide, a GP IIb/IIIa antagonist, decreased the VI to the level of MB-CON in these groups $(P<0.05$; Fig. $7 \mathrm{~B})$, suggesting that the binding between MB-cRGD and GP IIb/IIIa receptor was substantial inhibited by Eptifibatide. The VI of MB-cRGD in the testing group was also correlated with GP IIb/IIIa coverage of the endothelium and expression in plaques (Fig. 7C, D), as well as with vulnerability index and NC/FC (Fig. 7E, F).

\section{Discussion}

The results of this study demonstrate that GP $\mathrm{IIb} / \mathrm{III}$ receptors on activated platelets adhered to and aggregated on the endothelium can serve as biomarkers for plaques that can be identified and quantified ultrasonically. Specifically, it was shown that UMI with MBs targeted to GP IIb/IIIa receptors can detect atherosclerosis and high-risk plaques.

Platelet aggregation and thrombosis of the arterial endothelium play a pivotal role in the pathogenesis of plaque destabilization [11]. In addition, we demonstrated that activated platelets were frequently recruited and adhered to each other (Fig. 3Ah) as well as to the injured endothelial surface in high-risk plaques (Fig. 3Cb), in agreement with previous report [11]. The interaction is mainly mediated by integrin aIIb $\beta 3$, vWF, fibrinogen, fibrin, and/or fibronectin [34-36], suggesting that the extent to which activated platelets adhered to the endothelial surface of atherosclerotic plaques was positively associated with the degree of plaque instability. This was supported by the finding that fluorescence-labeled platelets adhered to the endothelium of atherosclerotic lesions and GP IIb/IIIa coverage of the endothelium were correlated with both NC/FC and vulnerability index. Finally, results from the in vitro study suggest that the inflammatory reaction leading to the increased expression of endothelial vWF initiated platelet activation/aggregation, resulting in the high expression of GP IIb/IIIa on activated and adhered platelets. Thus, these GP IIb/IIIa receptors could be a biomarker for vulnerable plaques that signals advanced atherosclerotic lesions, and their quantification could be used to evaluate atherosclerotic progression and vulnerability.

Several studies have examined the feasibility of targeting the GP IIb/IIIa of activated platelets using different agents in vitro and in vivo [37-39]. Recently, a non-specific anti-GP IIb/IIIa chimeric monoclonal antibody, abciximab; and a specific monoclonal antibody against ligand-induced binding sites were used successfully for the molecular imaging of thrombosis $[39,40]$. However, the noncovalent streptavidin-biotin interactions in these agents have immunogenic side effects; abciximab interferes with the GP IIb/IIIa receptor and prevents platelet aggregation and thrombus formation, which can lead to bleeding [41], thereby limiting its clinical applications. In contrast, the risk of bleeding associated with RGD is much lower due to the short duration of GP IIb/IIIa receptors blocking by this peptide [42]. In addition, RGD peptides and their analogs, which can be readily bound covalently to contrast agents, have a high affinity for GP IIb/IIIa [20, 24] and have been widely used to probe integrin function in various biological systems [24, 43], making them ideal molecular probes for targeting GP IIb/IIIa. A 30-fold higher binding affinity for GP IIb/IIIa is exhibited by cRGD than the linear peptide [21, 22], which is particularly useful under conditions of rapid blood flow that occur in atherosclerotic arteries. 
A
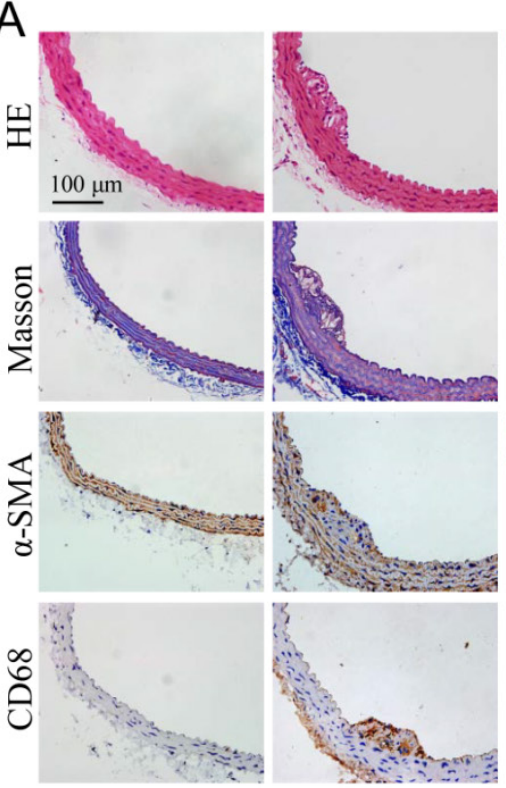

C57BL/6

+chow

F

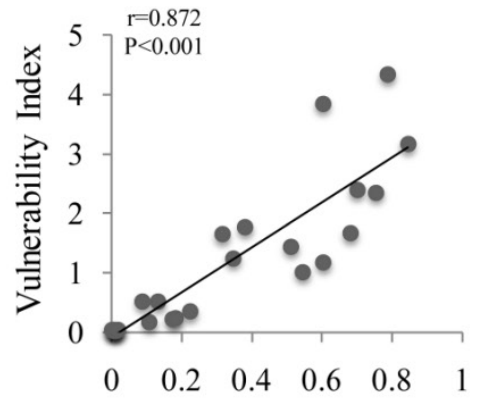

GP IIb/IIIa coverage

of the endothelium (\%)
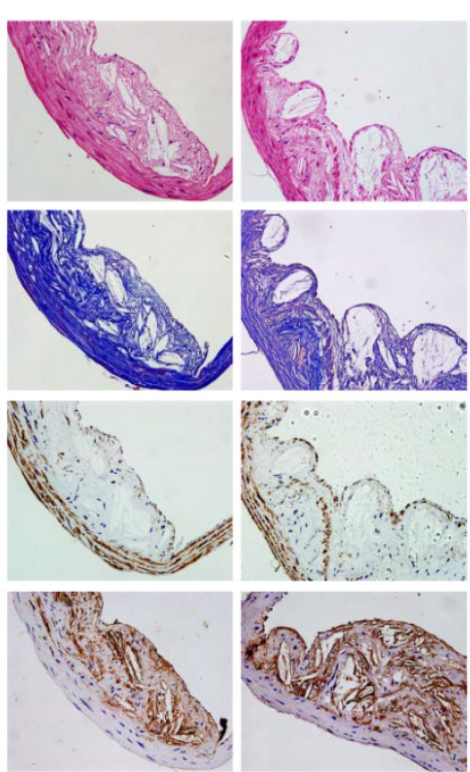

ApoE-/-

+chow

G

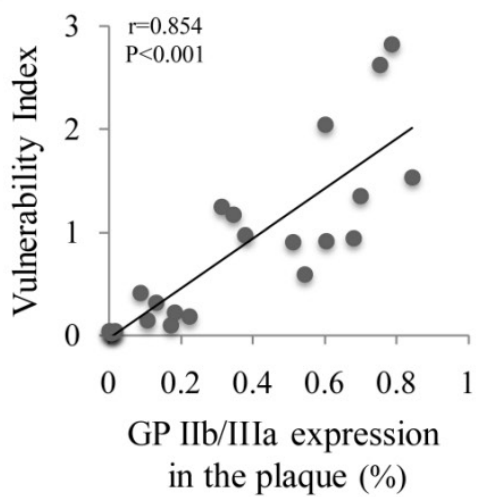

B

Plaque SMC content

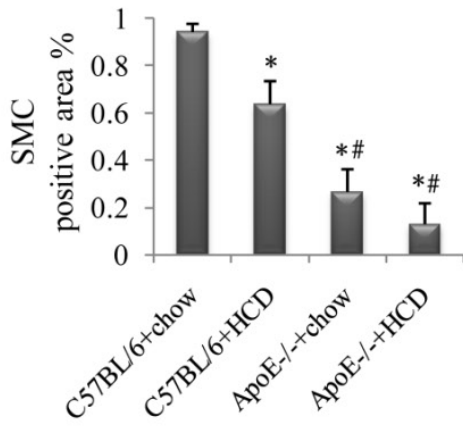

\section{Plaque macrophage}
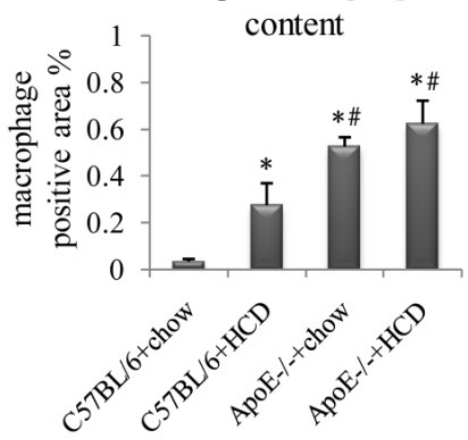

D

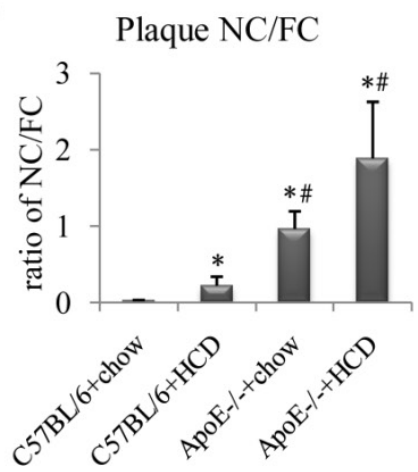

$\mathrm{H}$

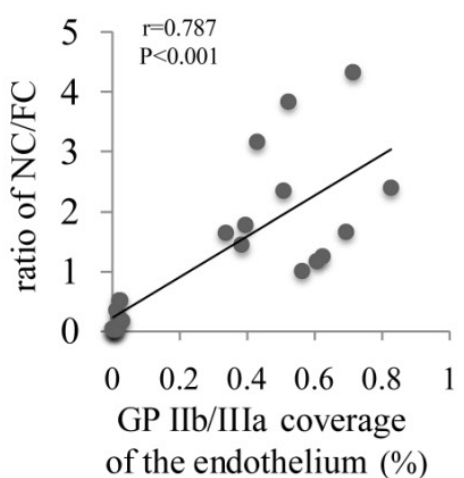

I

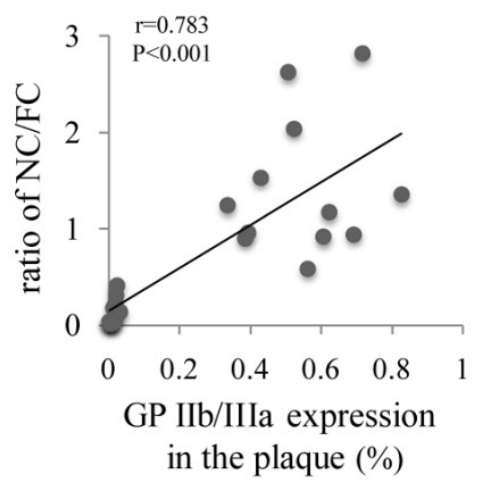

$E$

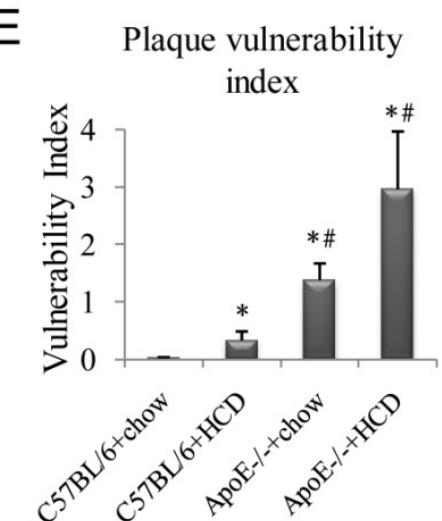

Figure 5: Histopathologic examination of atherosclerotic plaques. (A) Representative images of aortic tissue as visualized by hematoxylin and eosin and Masson's trichrome staining, or labeled with antibodies against $\alpha$-SMA and CD68. Experimental groups are as described in the Figure 2 legend. Quantitative analyses of (B) total plaque SMCs and (C) macrophage content are shown ( $n=6$ per group). (D) Plaque NC/FC and (E) vulnerability index were calculated. Correlations between plaque vulnerability index and (F) GP Ilb/llla coverage of the endothelium and (G) GP Ilb/llla expression in the plaque, and between plaque NC/FC and (H) GP Ilb/llla coverage of the endothelium and (I) GP Ilb/llla expression in the plaque were determined. 
A

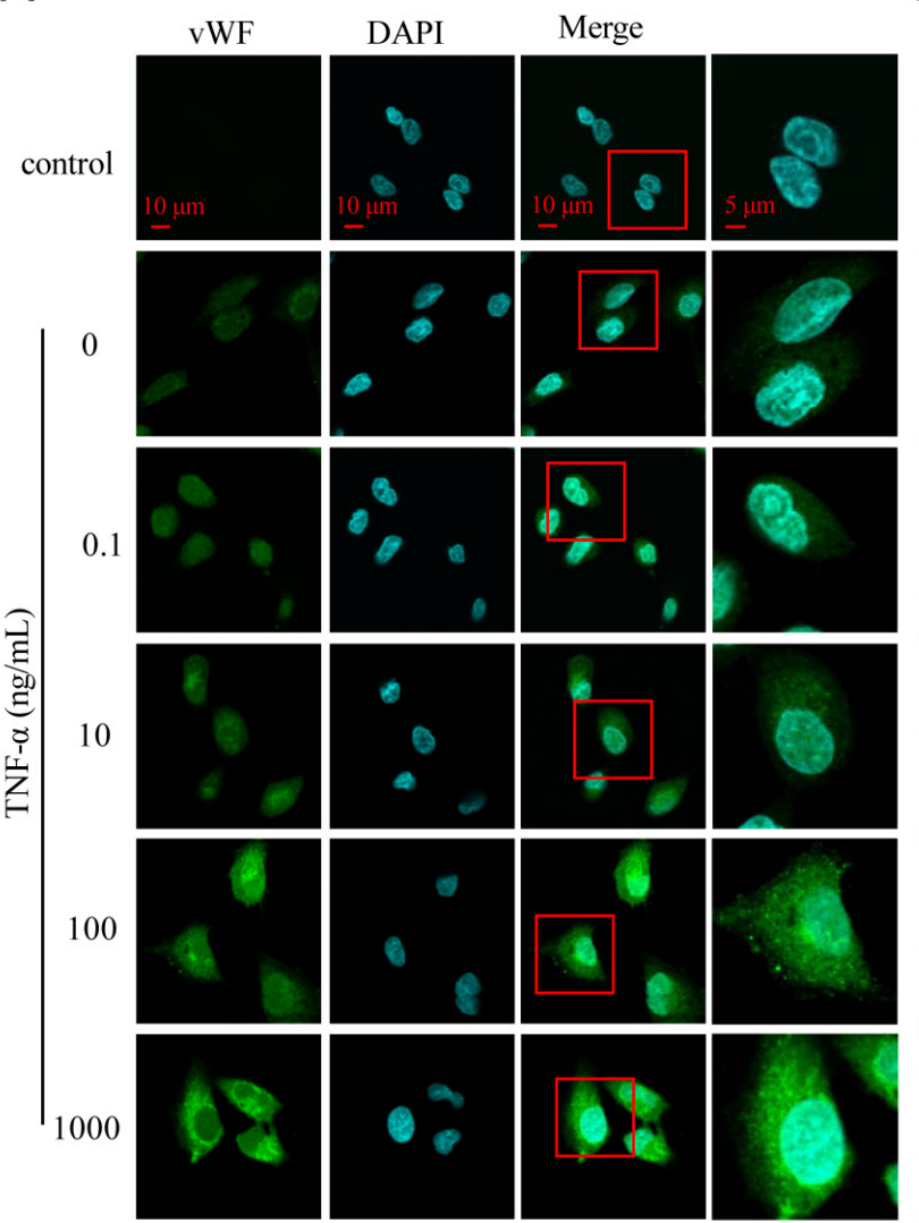

C

Platelet GP IIb/IIIa
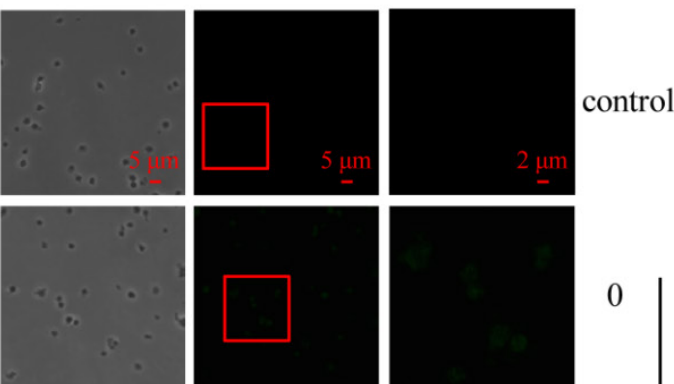

0
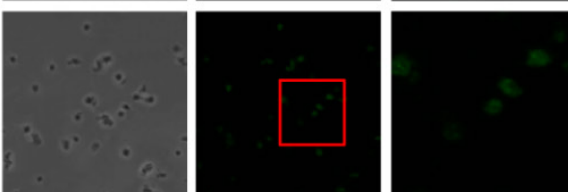

0.1
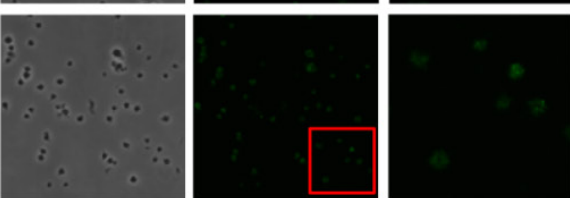

$\sum$
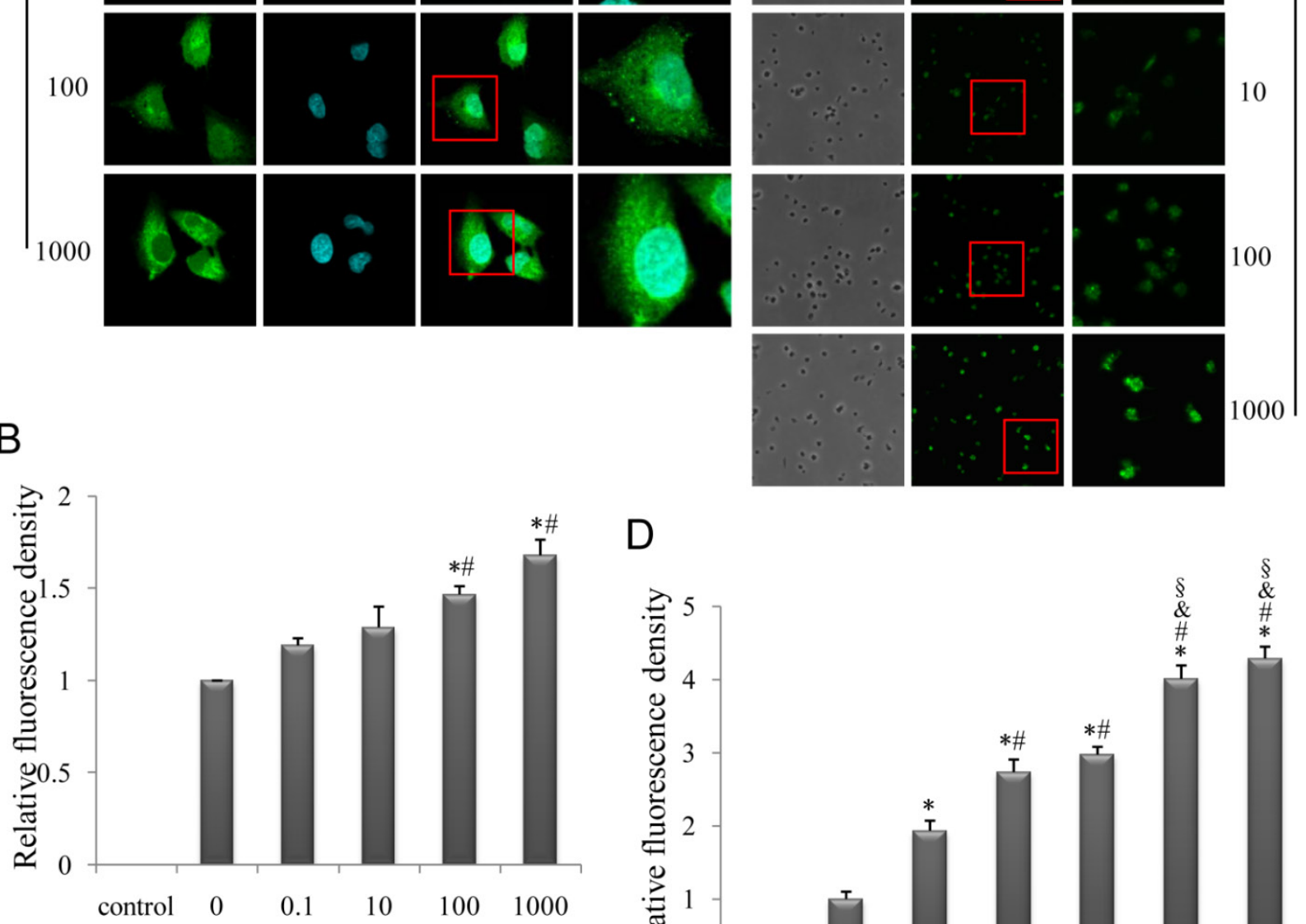

TNF $\alpha$ concentrations $(\mathrm{ng} / \mathrm{mL})$

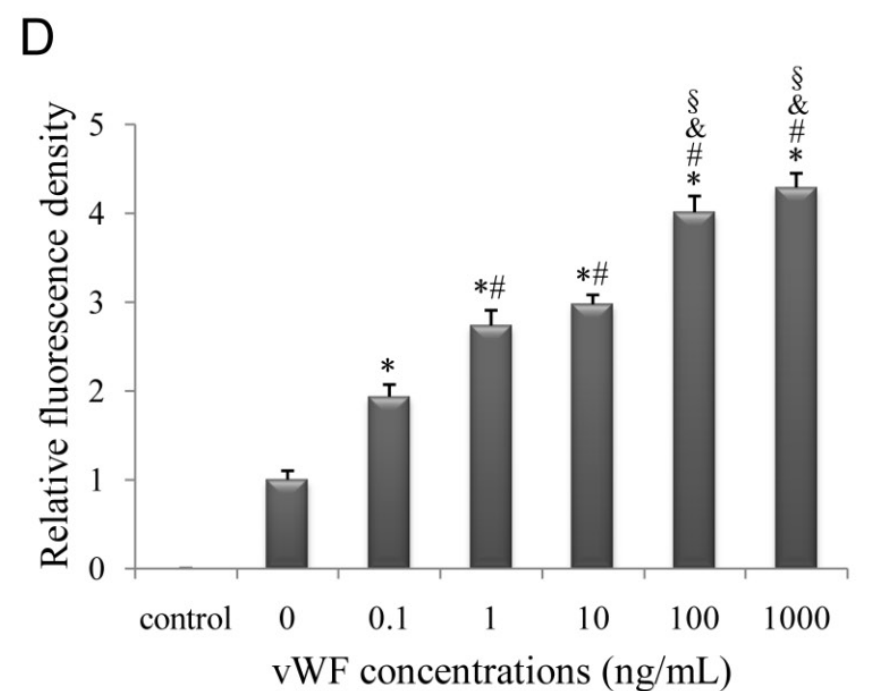

Figure 6: TNF- $\alpha$ increases endothelial vWF expression while GP Ilb/IIla expression on platelets is induced by vWF. (A, C) Representative confocal images of HUVECs (A) and platelets (C); HUVECs and platelets were stimulated with various concentrations of TNF- $\alpha$ and vWF, respectively, and evaluated for vWF and GP Ilb/llla expression, respectively. (B, D) Quantitative analysis of relative fluorescence signal intensity of vWF (B) and GP Ilb/Illa (D). Results are expressed as mean \pm standard deviation. $* P<0.05$ vs. $0 \mathrm{ng} / \mathrm{ml}, \# P<0.05 \mathrm{vs} .0 .1 \mathrm{ng} / \mathrm{ml}, \& P<0.05 \mathrm{vs}$. $1 \mathrm{ng} / \mathrm{ml}, \$ P<0.05 \mathrm{vs} .10 \mathrm{ng} / \mathrm{ml}$. 
A
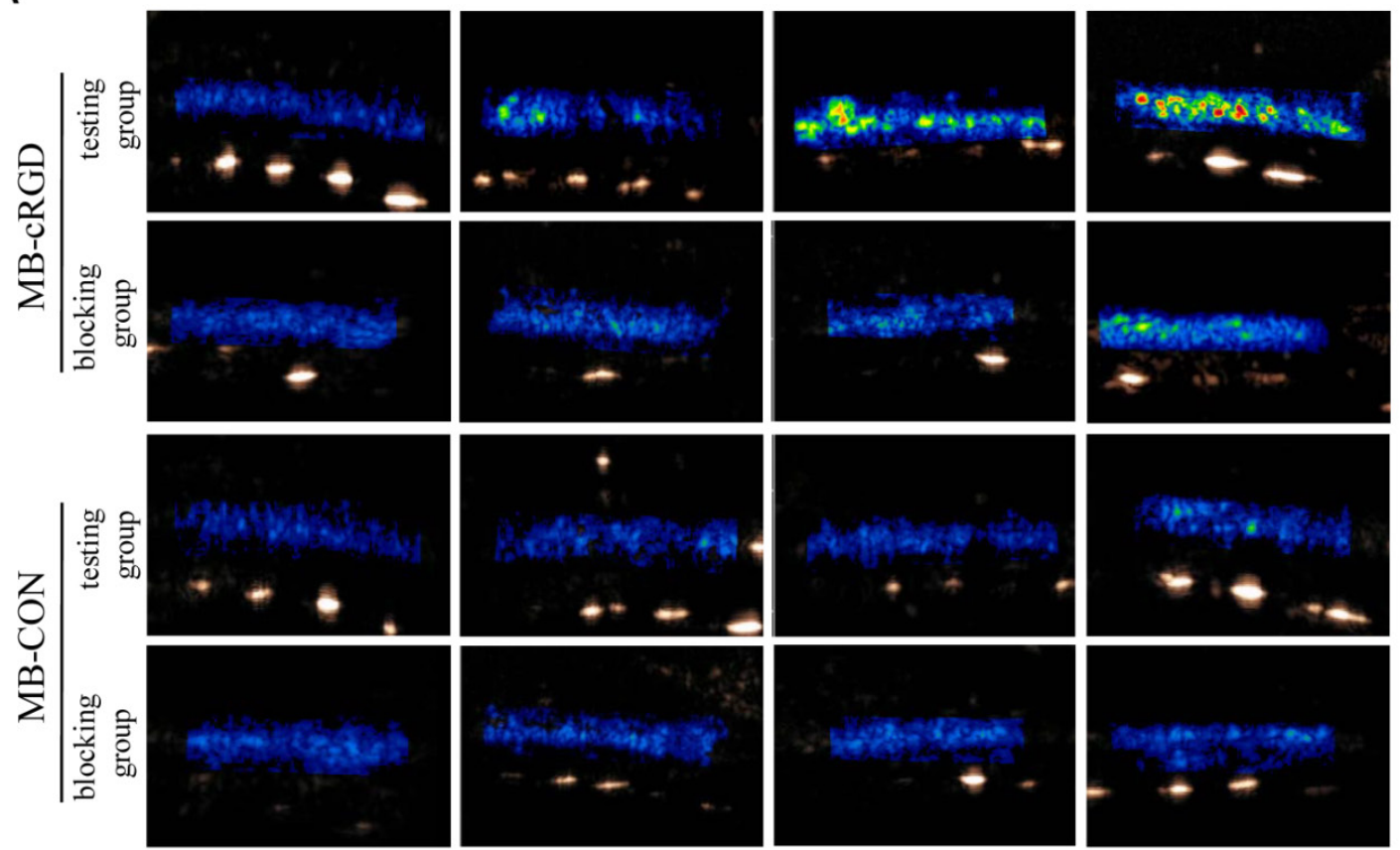

C57BL/6+chow

C57BL/6+HCD

ApoE-/-+chow

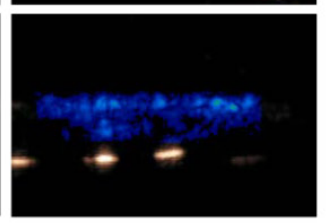

ApoE-/-+HCD

B

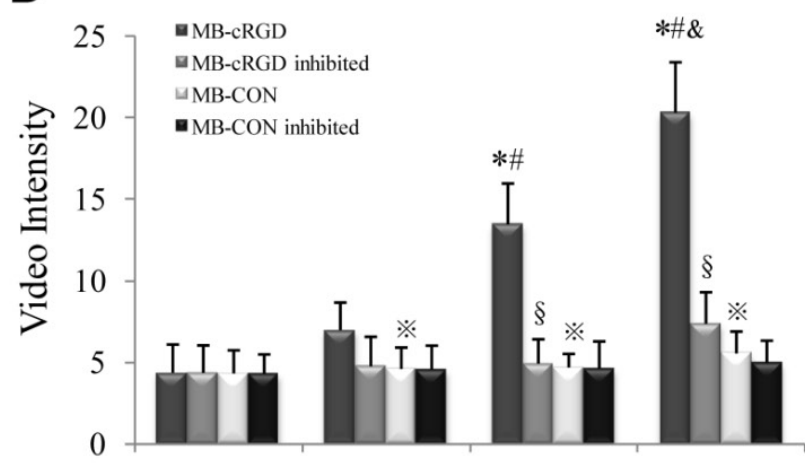

C

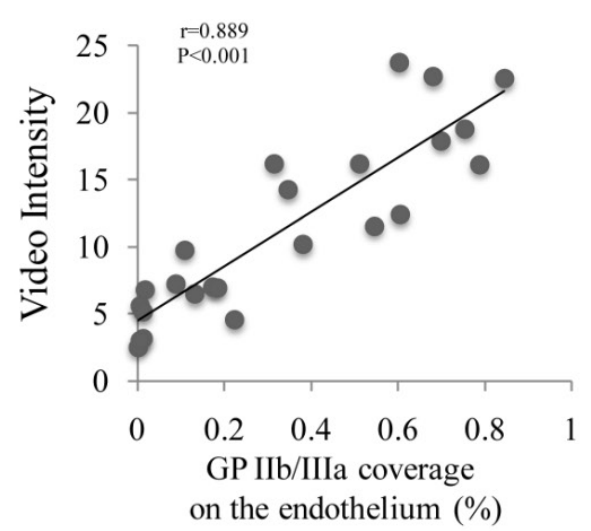

D

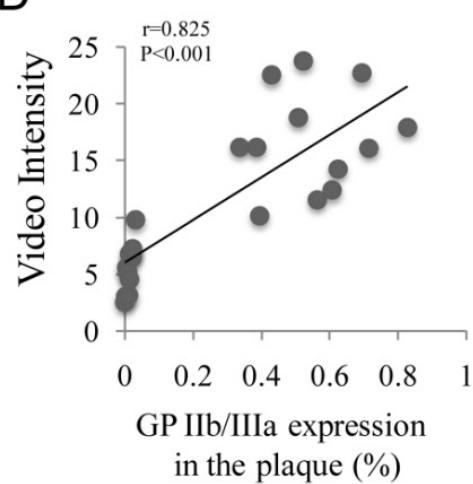

$E$

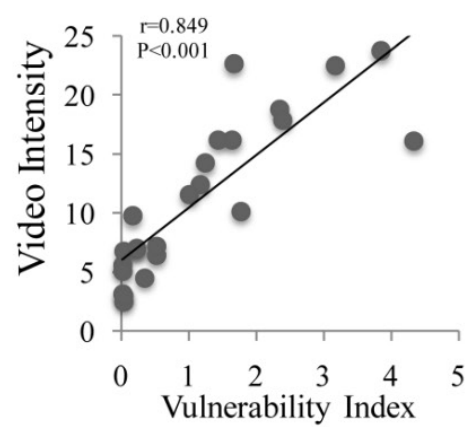

$\mathrm{F}$

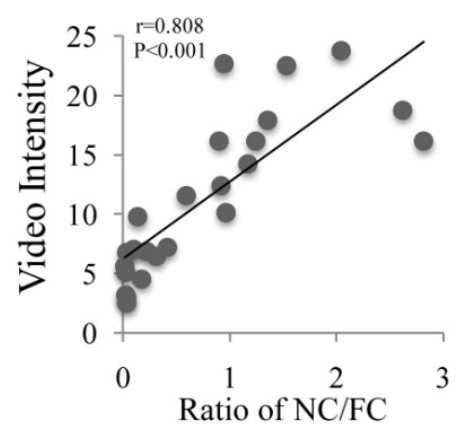

Figure 7: UMI of atherosclerosis in the test and inhibitor-treated mice and correlation with histopathologic indicators. (A) Background-subtracted, color-coded images were obtained $10 \mathrm{~min}$ after injection of a MB-cRGD or negative control (MB-CON) peptides. Experimental groups are as described in the Figure 2 legend. (B) The VI of MB-CRGD and MB-CON in the test mice and those pre-treated with eptifibatide was quantified ( ${ }^{*} P<0.05, M B-C R G D$ vs. MB-CON; $\$ P<0.05$ MB-cRGD inhibited vs. MB-cRGD; $n=6$ per group). Correlations between the VI of MB-cRGD in the test group and (C) GP IIb/llla coverage of the endothelium, (D) GP Ilb/llla expression in plaques, (E) plaque vulnerability index, and (F) NC/FC are shown. 
The present study found robust correlations between the VI of MB-cRGD on plaques and plaque vulnerability indicators, indicating that UMI with MBs targeted to GP IIb/IIIa receptors is capable of detecting atherosclerosis and high-risk plaques. MB-cRGDs mainly target GP IIb/IIIa receptors present on the exposed endothelial cell and thrombus surface, since MBs do not leave the intravascular space or enter the thrombus entirely. Moreover, GP $\mathrm{IIb} / \mathrm{III}$ a coverage of the endothelium was correlated with expression in plaques, and therefore provides an accurate readout of GP IIb/IIIa expression on the whole plaque, serving as an indicator of plaque vulnerability. The correlation between GP IIb/IIIa coverage of the endothelium and NC/FC as well as vulnerability index also provides evidence in support of this possibility.

Fluorescent signals similar to those from fluorescently labeled platelets were observed in the sub-endothelial lipid center. One explanation for this is that platelets aggregated at the site of injury and neovascularization via aIIb $\beta 3$ [44]; alternatively, fluorescently labeled platelets may have leaked from the lipid core through a ruptured vessel. The green spots within atherosclerotic plaques in the negative control mice may also be attributable to the leakage of fluorescent-dye, since the effect of autofluorescence was eliminated. Local VI accumulation in the aorta of ApoE ${ }^{-/-}+\mathrm{HCD}$ mice administered $\mathrm{MB}-\mathrm{CON}$ may have resulted from the nonspecific attachment and/or endocytosis of MB-CON by activated leukocytes or the entrapment of MB-CON by fibrin mesh [45].

It was reported that the size variation of platelet is large (the diameter of the platelet is ranging from $1 \sim 8 \mu \mathrm{m}$ under transmission EM) [32], which is in agreement with our findings in this study (Fig. 3Ca). To confirm our judgment, we further observed the microparticles in the platelets by using transmission EM (Fig. 3Cc and d). We should pay attention to that MB-cRGD not only binds to GP IIb/IIIa but also other integrins, such as av $\beta 3, \operatorname{av} \beta 1$, and $\operatorname{av} \beta 6$. Although their expression on activated platelets is much lower than that of GP IIb/IIIa [12, 46], they may contribute to the enhanced ultrasound signal from MB-cRGD, suggesting a limitation for the specificity of MB-cRGD to detect GP IIb/IIIa in activated platelets. MB-GP $\mathrm{IIb} / \mathrm{III}$ specific antibody may overcome this limitation, but it likely produces immunogenic side effects. In addition, our previous studies showed that MB-cRGD has a high capacity for binding at a continuous shear stress of $8 \mathrm{dyn} / \mathrm{cm}^{2}[24,25]$. Although it did not accurately mimic hemodynamics in atherosclerotic lesions in vivo, a high binding affinity was achieved in the current atherosclerotic model, in which binding may be enhanced during the diastolic phase between pulses when shear stress is markedly altered [47]. Furthermore, similar to previous studies, only a small fraction of the atherosclerotic plaques that were generated could be defined as vulnerable plaques, that could cause severe cardiovascular events; however, plaque vulnerability was observed in many mice, especially in those that were ApoE-deficient and on an HCD, as evidenced by instability indicators associated with atherosclerotic vulnerability [29]. In addition, it is possible that the sonication that was applied to disrupt MBs potentially destroyed the tissue, particularly the nutrient vessels of atherosclerotic plaques; however, there was no evidence of this at a mechanical index of 1.0. Indeed, the threshold mechanical index for UMI that can lead to tissue and/or microvessel destruction has yet to be established.

\section{Conclusions}

GP IIb/IIIa of activated platelets on the plaque endothelium is a novel, reliable biomarker for advanced atherosclerotic lesions that can be used to identify plaque instability by noninvasive molecular imaging and assist in the prevention of acute cardiovascular events.

\section{Supplementary Material}

Table S1, Figures S1-S2.

http://www.thno.org/v05p0418s1.pdf

\section{Abbreviations}

UMI: ultrasound molecular imaging; $\mathrm{MB}$ : microbubble; $\mathrm{MB}-\mathrm{CON}$ : negative control microbubble; MB-cRGD: cyclic RGD-modified microbubble; vWF: von Willebrand factor; GP: glycoprotein; RGD: Arg-Gly-Asp; ApoE-/-: apolipoprotein E-deficient; HCD: hypercholesterolemic diet; PBS: phosphate-buffered saline; EM: electron microscopy; a-SMA: a-smooth muscle actin; EM: electron microscopy; NC/FC: necrotic center/fiber cap ratio; TNF-a: tumor necrosis factor a; HUVEC: human umbilical vein endothelial cells; VI: video intensity; RBC: red blood cells;

\section{Acknowledgments}

This study was supported by grants from the National Basic Research Program of China (973 Program; no. 2013CB733804), the Team Program of Natural Science Foundation of Guangdong Province, China (no. S2011030003134), and the National Natural Science Foundation of China (nos. 81227801 and 81271640 to J.B.). 


\section{Competing Interests}

The authors have declared that no competing interest exists.

\section{References}

1. Waxman S, Ishibashi F, Muller JE. Detection and treatment of vulnerable plaques and vulnerable patients: novel approaches to prevention of coronary events. Circulation. 2006; 114: 2390-411.

2. Fleg JL, Stone GW, Fayad ZA, et al. Detection of high-risk atherosclerotic plaque: report of the NHLBI Working Group on current status and future directions. JACC Cardiovasc Imaging. 2012; 5: 941-55.

3. Lindner JR. Molecular imaging of cardiovascular disease with contrast-enhanced ultrasonography. Nat Rev Cardiol. 2009; 6: 475-81.

4. McCarty OJ, Conley RB, Shentu W, et al. Molecular imaging of activated von Willebrand factor to detect high-risk atherosclerotic phenotype. JACC Cardiovasc Imaging. 2010; 3: 947-55.

5. Liu Y, Davidson BP, Yue Q, et al. Molecular imaging of inflammation and platelet adhesion in advanced atherosclerosis effects of antioxidant therapy with NADPH oxidase inhibition. Circ Cardiovasc Imaging. 2013; 6: 74-82.

6. Kaufmann BA, Sanders JM, Davis C, et al. Molecular imaging of inflammation in atherosclerosis with targeted ultrasound detection of vascular cell adhesion molecule-1. Circulation. 2007; 116: 276-84.

7. Kaufmann BA, Carr CL, Belcik JT, et al. Molecular imaging of the initial inflammatory response in atherosclerosis: implications for early detection of disease. Arterioscler Thromb Vasc Biol. 2010; 30: 54-9.

8. Liu H, Wang $\mathrm{X}$, Tan KB, et al. Molecular imaging of vulnerable plaques in rabbits using contrast-enhanced ultrasound targeting to vascular endothelial growth factor receptor-2. J Clin Ultrasound. 2011; 39: 83-90.

9. $\mathrm{Lu} \mathrm{Y}, \mathrm{Wei} \mathrm{J}$ Shao $\mathrm{Q}$ et al. Assessment of atherosclerotic plaques in the rabbit abdominal aorta with interleukin-8 monoclonal antibody-targeted ultrasound microbubbles. Mol Biol Rep. 2013; 40: 3083-92.

10. Huo Y, Schober A, Forlow SB, et al. Circulating activated platelets exacerbate atherosclerosis in mice deficient in apolipoprotein E. Nat Med. 2003; 9: 61-7.

11. Jackson SP. Arterial thrombosis--insidious, unpredictable and deadly. Nat Med. 2011; 17: 1423-36.

12. Wagner CL, Mascelli MA, Neblock DS, et al. Analysis of GPIIb/IIIa receptor number by quantification of 7E3 binding to human platelets. Blood. 1996; 88: 907-14.

13. Aukrust P, Halvorsen $\mathrm{B}$, Ueland $\mathrm{T}$, et al. Activated platelets and atherosclerosis. Expert Rev Cardiovasc Ther. 2010; 8: 1297-307.

14. Dymicka-Piekarska V, Kemona H, Butkiewicz A, et al. Platelets and platelet microparticles glycoprotein $\mathrm{Ilb} / \mathrm{III}$ a complex in patients with unstable angina. Pol Merkur Lekarski. 2005; 18: 9-12.

15. Yakushkin VV, Zyuryaev IT, Khaspekova SG, et al. Glycoprotein IIb-IIIa content and platelet aggregation in healthy volunteers and patients with acute coronary syndrome. Platelets. 2011; 22: 243-51.

16. Chakhtoura EY, Shamoon FE, Haft JI, et al. Comparison of platelet activation in unstable and stable angina pectoris and correlation with coronary angiographic findings. Am J Cardiol. 2000; 86: 835-9.

17. Singh N, Gemmell CH, Daly PA, et al. Elevated platelet-derived microparticle levels during unstable angina. Can J Cardiol. 1995; 11: 1015-21.

18. Ikuta T, Naruko T, Ikura Y, et al. Immunolocalization of platelet glycoprotein $\mathrm{IIb} / \mathrm{III}$ and P-selectin, and neutrophil-platelet interaction in human coronary unstable plaques. Int J Mol Med. 2005; 15: 573-7.

19. Lam SC, Plow EF, Ginsberg MH. Platelet membrane glycoprotein IIb heavy chain forms a complex with glycoprotein IIIa that binds Arg-Gly-Asp peptides. Blood. 1989; 73: 1513-8.

20. Ruoslahti E, Pierschbacher MD. New perspectives in cell adhesion: RGD and integrins. Science. 1987; 238: 491-7.

21. Bogdanowich-Knipp SJ, Chakrabarti S, Williams TD, et al. Solution stability of linear vs. cyclic RGD peptides. J Pept Res. 1999; 53: 530-41.

22. Bogdanowich-Knipp SJ, Jois DS, Siahaan TJ. The effect of conformation on the solution stability of linear vs. cyclic RGD peptides. J Pept Res. 1999. 53. 523-9.

23. Choi $\mathrm{H}$, Aboulfatova $\mathrm{K}$, Pownall HJ, et al. Shear-induced disulfide bond formation regulates adhesion activity of von Willebrand factor. J Biol Chem. 2007; 282: 35604-11.

24. Hu G, Liu C, Liao $Y$, et al. Ultrasound molecular imaging of arterial thrombi with novel microbubbles modified by cyclic RGD in vitro and in vivo. Thromb Haemost. 2012; 107: 172-83.

25. Wu W, Wang $Y$, Shen $S$, et al. In Vivo Ultrasound Molecular Imaging of Inflammatory Thrombosis in Arteries With Cyclic Arg-Gly-Asp-Modified
Microbubbles Targeted to Glycoprotein IIb/IIIa Invest Radiol. 2013;48:803-812

26. Kobayashi T, Tahara $\mathrm{Y}$, Matsumoto M, et al. Roles of thromboxane $\mathrm{A}(2)$ and prostacyclin in the development of atherosclerosis in apoE-deficient mice. J Clin Invest. 2004; 114: 784-94.

27. Johnson JL, Devel L, Czarny B, et al. A selective matrix metalloproteinase-12 inhibitor retards atherosclerotic plaque development in apolipoprotein E-knockout mice. Arterioscler Thromb Vasc Biol. 2011; 31: 528-35.

28. Alexander MR, Moehle CW, Johnson JL, et al. Genetic inactivation of IL-1 signaling enhances atherosclerotic plaque instability and reduces outward vessel remodeling in advanced atherosclerosis in mice. J Clin Invest. 2012; 122: 70-9.

29. Shiomi M, Ito T, Hirouchi Y, et al. Fibromuscular cap composition is important for the stability of established atherosclerotic plaques in mature WHHL rabbits treated with statins. Atherosclerosis. 2001; 157: 75-84.

30. Guo T, Chen WQ, Zhang C, et al. Chymase activity is closely related with plaque vulnerability in a hamster model of atherosclerosis. Atherosclerosis. 2009; 207: 59-67

31. Wu J, Leong-Poi H, Bin J, et al. Efficacy of contrast-enhanced US and magnetic microbubbles targeted to vascular cell adhesion molecule-1 for molecular maging of atherosclerosis. Radiology. 2011; 260: 463-71.

32. Hulander $M$, Lundgren $A$, Faxalv $L$, et al. Gradients in surface nanotopography used to study platelet adhesion and activation. Colloids Surf B Biointerfaces. 2013: 110: 261-9.

33. Theilmeier G, Michiels C, Spaepen E, et al. Endothelial von Willebrand factor recruits platelets to atherosclerosis-prone sites in response to hypercholesterolemia. Blood. 2002; 99: 4486-93.

34. Ruggeri ZM, Bader R, de Marco L. Glanzmann thrombasthenia: deficient binding of von Willebrand factor to thrombin-stimulated platelets. Proc Natl Acad Sci U S A. 1982; 79: 6038-41.

35. Bennett JS, Vilaire G. Exposure of platelet fibrinogen receptors by ADP and epinephrine. J Clin Invest. 1979; 64: 1393-401.

36. Ni H, Denis CV, Subbarao S, et al. Persistence of platelet thrombus formation in arterioles of mice lacking both von Willebrand factor and fibrinogen. J Clin Invest. 2000; 106: 385-92.

37. Unger EC, McCreery TP, Sweitzer RH, et al. In vitro studies of a new thrombus-specific ultrasound contrast agent. Am J Cardiol. 1998; 81: 58G-61G.

38. Takeuchi M, Ogunyankin $\mathrm{K}$, Pandian NG, et al. Enhanced visualization of intravascular and left atrial appendage thrombus with the use of a thrombus-targeting ultrasonographic contrast agent (MRX-408A1): In vivo experimental echocardiographic studies. J Am Soc Echocardiogr. 1999; 12: 1015-21.

39. Alonso A, Della Martina A, Stroick M, et al. Molecular imaging of human thrombus with novel abciximab immunobubbles and ultrasound. Stroke. 2007; 38: 1508-14

40. Wang $X$, Hagemeyer CE, Hohmann JD, et al. Novel single-chain antibody-targeted microbubbles for molecular ultrasound imaging of thrombosis: validation of a unique noninvasive method for rapid and sensitive detection of thrombi and monitoring of success or failure of thrombolysis in mice. Circulation. 2012; 125: 3117-26.

41. Peter K, Kohler B, Straub A, et al. Flow cytometric monitoring of glycoprotein $\mathrm{Ib} / \mathrm{III}$ blockade and platelet function in patients with acute myocardial infarction receiving reteplase, abciximab, and ticlopidine: continuous platelet inhibition by the combination of abciximab and ticlopidine. Circulation. 2000; 102: 1490-6.

42. Coller BS. Anti-GPIIb/IIIa drugs: current strategies and future directions. Thromb Haemost. 2001; 86: 427-43.

43. Burtea C, Laurent S, Murariu O, et al. Molecular imaging of alpha v beta3 integrin expression in atherosclerotic plaques with a mimetic of RGD peptide grafted to Gd-DTPA. Cardiovasc Res. 2008; 78: 148-57.

44. van Lammeren GW, Pasterkamp G, de Vries JP, et al. Platelets enter atherosclerotic plaque via intraplaque microvascular leakage and intraplaque hemorrhage: a histopathological study in carotid plaques. Atherosclerosis. 2012; 222: 355-9

45. Lindner JR Cogoins MP Kaul $S$, et al Microbubble persistence in the microcirculation during ischemia/reperfusion and inflammation is caused by integrin- and complement-mediated adherence to activated leukocytes. Circulation. 2000; 101: 668-75.

46. Kasirer-Friede A Kahn ML Shattil SJ. Platelet integrins and immunoreceptors. Immunol Rev. 2007; 218: 247-64.

47. Palm-Meinders IH, Box FM, de Craen AJ, et al. Diastolic wall shear stress in the internal carotid artery is associated with different cardiovascular risk factors than systolic wall shear stress. Cerebrovasc Dis. 2009; 28: 185-90. 